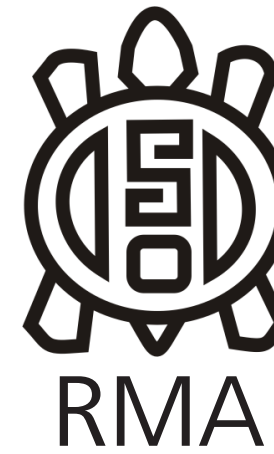

Dossier

Wichí: La palabra

\title{
Las palabras de la moto. Acercamiento al mundo wichí a través de la mecánica
}

\author{
The motorcycle's words. Approaching the Wichi world \\ through mechanics
}

\author{
Alberto Preci* \\ *UMR 8586 Prodig - Université Paris 1 Panthéon-Sorbonne. \\ E-mail: Alberto.Preci@univ-paris1.fr
}

\begin{abstract}
Resumen
Haciendo hincapié en un estudio de la lengua wichí, el objetivo de este artículo es estudiar las motos, y aun más su adopción y adaptación verbal, en cuanto vectores para avanzar en la comprensión de la aculturación léxica de los wichís en un marco de relaciones interétnicas. El estudio de la lengua se constituye como una etapa previa e imprescindible para entender la complejidad actual del mundo wichí y la manera en que los indígenas se relacionan con lo mecánico. Este artículo se basa sobre un análisis de los términos que los jóvenes wichís del Chaco formoseño que participan de unos cursos para devenir mecánicos, traducen, integran y adaptan para apropiarse no solamente de un vocabulario técnico, sino también de las mismas motos. Los resultados previos de esta investigación que aquí se presentan atestiguan de las diversas estrategias lexicales que los jóvenes wichís desarrollan en la medida que elementos de distintas culturas y saberes se entremezclan. En este sentido, investigar las palabras de las motos se vuelva una herramienta útil y pertinente para avanzar tanto en el estudio de la lengua de los jóvenes wichís, como de su cultura.
\end{abstract}

Palabras clave: Lengua wichí; Motos; Mecánica; Aculturación léxica; Vocabulario técnico.

\begin{abstract}
Stressing a study of the Wichi language, the objective of this article is to study the motorcycles, and even more their adoption and verbal adaptation, as vectors for advancing the understanding of lexical Wichi acculturation within a framework of inter-ethnic relations. The study of the language is constituted as a previous and essential stage to understand the current complexity of the Wichi world and the way in which they relate to the mechanical. This article is based on an analysis of the terms that the young Wichi of the Chaco region of Formosa, who are taking part in classes to become mechanics, translate, integrate and adapt in order to appropriate not only a technical vocabulary, but also the motorcycles themselves. The findings of this research testify to the diverse lexical strategies that the young Wichis develop as elements from different cultures and knowledge intermingle. In this sense, investigating the motorcycles words becomes a useful and pertinent tool to advance in the study of the language of the young Wichis, as well as their culture.
\end{abstract}

Keywords: Wichí language; Motorcycles; Mechanics; Lexical acculturation; Technical vocabulary.

Una moto tirada no sirve. Y el Chaco está lleno de motos tiradas. Por eso, a pesar de su reciente aparición, sería legitimo hacer una arqueología de estas máquinas, cuyas piezas ya constituyen un elemento del paisaje chaqueño, tanto urbano como rural. Pero, al contrario de lo que se podría pensar, esos residuos mecánicos diseminados en el monte, al lado de cada vivienda, a las orillas de la ruta o en los pueblos no son basura, ni son los restos de una modernidad prometida, que en la región chaqueña siempre está por llegar, sin arrancar nunca.

En realidad, esas motos solamente están detenidas, paradas, en espera. Ahí están y estarán por el tiempo necesario para que sus dueños entiendan la traba que las vuelve inútiles, cómo resolverla y qué tipo de repuesto se requiere para arreglarlas. Pero para eso, hace falta tiempo, y sus dueños necesitan observarlas, entenderlas y apropiárselas para luego poder arreglarlas, resucitarlas y reutilizarlas. Retomando una expresión utilizada localmente, se puede decir que se necesita el tiempo necesario "para hacerlas que anden de vuelta". Este lapso de tiempo sin definir, que puede ser días o años, y durante del cual pueden aparecer nuevas y otras motos, corresponde a ese largo proceso de introducción, 
de adopción y de comprensión de las motos que se está actualmente desarrollando en el Chaco, allí donde las motos constituyen un medio de transporte relativamente nuevo e indudablemente fundamental.

\section{Entre traducción y apropiación cultural de las motos}

Entre los indígenas chaqueños, y sobre todo entre los wichís, desde el comienzo de los años 2000 las motos se han popularizado de manera contundente, contribuyendo a reducir los tiempos de traslado mientras que se aceleran los intercambios y amplían las distancias que pueden recorrerse. Las dinámicas sociales, económicas, políticas, territoriales y de genero se renuevan desde la llegada masiva de las motos a la región. Todos estos cambios implican una reformulación parcial -aunque por eso no menos importante- tanto del espacio como del tiempo y, por ende, del "mundo" (Pina-Cabral 2014) wichí. Además de eso, la llegada de las motos trae consigo la necesidad de desarrollar un saber mecánico, que ciertamente se nutre de la herencia dejada por los misioneros anglicanos y sus talleres o por los equipos católicos de pastoral aborigen y sus aserraderos, pero que todavía es un saber incipiente.

Así estando las cosas, a lo largo de este artículo me enfocaré menos en los cambios que se están dando en el Chaco, que en estudiar de qué manera la moto se inscribe en la cotidianidad de los wichís a través de la lengua. Según Keith Basso (1967), gracias a su flexibilidad, la lengua registra los cambios quizás más sensiblemente que cualquier otro elemento de la cultura. Más precisamente, según el autor "es en el vocabulario donde se detectan con mayor facilidad estos cambios, ya sea debido a la evolución de la cultura o a los efectos del contacto intercultural" (Basso, 1967, p. 471). De hecho, tal como lo comenta Susana Susnik:

"Cuando los Chamacocos por primera vez vieron un avión, éste les evocaba el mítico ser alado y antropófago Anörxyt; al acostumbrarse al verlo sobrevolando su 'costa', el avión era ya una 'caja de hierro caminante'; al poder observarlo de cerca, el avión ya 'tenía ojos, cara' (ylys"); y cuando el avión era ya un medio normal de la comunicación norteña, la palabra castellana 'avión' introdújose definitivamente en el léxico chamacoco» (Susnik, 1969, p. 9).

Esta integración evolutiva no es propia de los Chamacocos y bien se adapta a los wichís. En este sentido, los frecuentes contactos entre representantes de la cultura dominante y grupos indígenas han tenido como consecuencia, entre otras cosas, la introducción de una serie de "nuevas realidades" -sean técnicas, máquinas, herramientas, materiales o alimentos- previamente desconocidas que, en mayor o menor medida, han sido integradas al mundo indígena (Dorais 1970). Esta forma de aculturación ha tenido su contrapartida lingüística: en la medida en que los indígenas han conservado su lengua, también han necesitado nombrar estas nuevas realidades. Por lo tanto, el léxico se ha transformado de acuerdo con los cambios culturales mientras el vocabulario se ha ajustado al nuevo contexto interétnico. De esa manera, ha tomado forma una "aculturación léxica" que, por los indígenas, implica la adopción de distintas estrategias: incorporar prestamos, implementar traducciones literales con innovaciones semánticas, ampliar los alcances semánticos de algunas palabras con las que ya se cuenta, acuñar expresiones descriptivas basadas en la descripción de algunas características físicas (Brown 1999). ${ }^{1}$

La aculturación ha sido largamente estudiada en varios momentos y con diversos enfoques, concentrándose en el contexto de relación interétnico. Asimismo, las estrategias adoptadas por distintos grupos indígenas han sido el objeto de análisis prolijos (Krohn 2016; García y García Molina 2016). En este articulo, se pone menos énfasis sobre el aspecto mutuo de la aculturación léxica para privilegiar la lengua wichí. ${ }^{2}$ Haciendo hincapié en esta lengua, propongo llevar adelante una etnografía de las motos para un mejor entendimiento de la complejidad del mundo wichí en la actualidad. De ahí, algunas preguntas empíricas guiarán mi análisis: ¿Como se habla de motos, de mecánica y de repuestos en lengua wichí? ¿En qué medida se traducen los términos castellanos para indicar una y otra pieza de un motor? ¿Y cómo se desarrolla la traducción? Demandas que, indirectamente, me llevan a preguntar ¿Qué lugar tienen las motos en el mundo wichí? ¿De qué manera los wichís, a través de su lengua, la integran a su mundo? Y, en definitiva, ¿qué rol tienen -siempre y cuando lo tengan- las motos en el cambio cultural que se está dando en el Chaco, donde objetos y actores foráneos no dejan de aparecer desde hace más de un siglo?

Si abordar esas cuestiones con algunas herramientas tomadas de la lingüística puede parecer una opción sorprendente y aun más tomar el caso de la "moto" como objeto de estudio puede resultar de los menos frecuentes, creo que los estudios de la lengua se constituyen como una etapa previa, o por lo menos ineludible, para entender el mundo wichí y la manera en la cual los wichís se relacionan con las motos y, más en general, con lo mecánico. Asimismo, estoy convencido que el

\footnotetext{
1 La expresión «aculturación léxica» hace referencia a los procesos de acomodación de una lengua ante nuevos conceptos y objetos encontrados, como resultado del contacto cultural (Brown, 1999). Desde un punto de vista etnológico, se refiere a los cambios que tienen lugar en un grupo cultural como resultado del contacto continuo con un grupo de otra cultura. Desde el punto de vista sociológico, apunta al proceso por el cual un individuo aprende las pautas de comportamiento, los modelos y las normas de un grupo a fin de ser aceptado.

2 Este "recorte" es debido a que el análisis de la presencia de prestamos en el castellano de la lengua wichí sobrepasa por mucho mis capacidades. Además, probablemente por falta de atención, no conozco estudios de este tipo sobre la lengua wichí, al estilo de lo que García (2016) propone sobre la presencia del cora en el castellano.
} 
mundo wichí ha experimentado, desde por lo menos un siglo, distintas "incursiones mecánicas" de las cuales las motos no son que el ultimo apéndice. ${ }^{3}$ Por eso, mis consideraciones entre motos, repuestos y palabras me llevarán a reflexionar sobre la construcción de un saber mecánico indígena a partir del estudio de un vocabulario específico, y más precisamente de las traducciones y de los términos que los wichís utilizan para indicar las distintas piezas que componen una moto y los repuestos necesarios para arreglarlas.

Basándome sobre un estudio de la apropiación del léxico mecánico o -parafraseando aquí una expresión de Rodrigo Montani (2017)- las "palabras de las motos", mi objetivo es entonces estudiar las motos, y aun más su adopción y adaptación verbal, en cuanto vectores para avanzar en la comprensión del mundo wichí. Así como las "palabras de dios" no son conceptos universales y no tienen ningún sentido evidente en la lengua wichí porque no encuentran una correspondencia en su entorno físico y aún menos en el cultural (Montani, 2017), lo mismo se puede decir de las palabras de las motos, donde expresiones como "culata", "cilindros", "colector de escape" o "muelle de contraste" no tienen mucho o, mejor dicho, no tienen ningún sentido intuitivo para los wichís. O quizás, como veremos, seguramente tienen algún sentido si es que los wichís logran traducir estas "palabras técnicas" en su lengua y, haciendo eso, terminan apropiándose lingüísticamente y culturalmente las motos y sus partes.

Esto dicho, quiero aclarar que estas páginas corresponden al intento de un geógrafo-ocasionado por un interés amateur en la materia- de movilizar herramientas propias de la lingüística y de la sociolingüística con el fin de infirmar o confirmar algunas hipótesis tentativas de investigación. Esas se enmarcan en un proyecto de investigación más amplio, y surgen de la voluntad de estudiar los cambios que se están dando hoy en día en del mundo wichí bajo el prisma de las motos. Persiguiendo este objetivo, mi reflexión se desarrollará en cuatros momentos. Luego de una breve presentación del contexto de mi investigación, me dedicaré a una etnografía de las motos y de su gente. Siguen unos comentarios sobre las modalidades de aprendizaje del saber mecánico, antes de presentar el conjunto de términos que nombran las piezas que constituyen una moto. Finalmente, haré unos intentos de interpretación del léxico mecánico que está tomando forma en la encrucijada de las lenguas.

\section{El mundo wichí en unas palabras}

\section{En el marco de un sistema extremadamente fluido y}

\footnotetext{
${ }^{3}$ Con eso, me refiero a los talleres y a los aserraderos cuya presencia es debida a la implantación de distintas misiones religiosas en el Chaco y, también, a la instalación temprana (segunda mitad del siglo XIX) de distintos ingenios azucareros situados en la zona pedemontana $\mathrm{y}$ otras "enclaves capitalistas en el medio de la selva" (Córdoba, Bossert, Richard 2015).
}

constantemente restructurado, distintos grupos wichís habitan un territorio relativamente vasto correspondiente a la llanura chaqueña entre los ríos Bermejo y Pilcomayo. En este trabajo, me enfocaré únicamente sobre la zona geográfica que se identifica como la cuenca baja del río Pilcomayo, lo que corresponde administrativamente hablando, al departamento Ramón Lista de la provincia argentina de Formosa, departamento que limita con el Paraguay. Esta zona se caracteriza por haber sido colonizada tardíamente, en comparación con la colonización más temprana del área del Bermejo, y por haber permanecido relativamente aislada hasta el fin del siglo XX en razón de la falta de accesibilidad y del escaso interés económico que representaba para las autoridades nacionales y provinciales.

A pesar de las diferentes trayectorias históricas que identifican a los grupos wichís según su localización, a lo largo de los años se ha mantenido una cierta cohesión territorial. Además, los wichís comparten unos rasgos culturales similares y hablan una misma lengua. No obstante, en razón de su autonomía, de su aislamiento y de las distancias, cada uno de esos grupos habla un dialecto diferente. ${ }^{4}$ Por eso, la diversidad constitutiva de la lengua wichí se vuelve una variable imprescindible para considerar a la hora de emprender un estudio del vocabulario de las motos que manejan los wichís del Pilcomayo formoseño. Efectivamente, los términos que cada hablante utiliza no son necesariamente válidos o legítimos por hablantes de otros grupos y, por ende, de otros dialectos. Dada esta diversidad intrínseca tanto de la cultura como de la lengua wichí, resulta imposible, o por lo menos sumamente complejo, desarrollar un análisis del vocabulario de la moto coherente y al mismo tiempo pertinente para todos los grupos. Como bien lo dice John Palmer, si toda investigación contribuye en forma acumulativa al conocimiento y a la comprensión del mundo wichi, "aunque exista consenso a grandes rasgos, siempre habrá disentimiento con respecto a los detalles, y viceversa" (2005, p. 2).

\section{Si bien previamente entretenían relaciones con otros}

\footnotetext{
${ }^{4}$ Varios lingüistas han propuesto unas subdivisiones de la lengua wichí en distintos dialectos sobre la base de un criterio territorial y lingüístico. Así pues, esos autores operan una distinción no solamente entre los dialectos hablados en las cuencas de los ríos Pilcomayo y Bermejo, sino también en función de la localización de los grupos que viven río arriba, cerca de las fuentes, o río abajo, cerca de las desembocaduras. A todo eso hay que agregar, una tercera opción que distingue entre los grupos históricamente asentados cerca del río y los que viven "monte adentro", también identificados como "montaraces" (Alvarsson 1988; Braunstein 2006; Montani 2017). Además de eso, la lengua wichí tal como se la habla actualmente es en buena medida el fruto de los distintos procesos histórico y sociales que han caracterizado la región, contribuyendo a cada vez más cambios e intercambios que no ocurrieron, ni ocurren de la misma manera a lo largo de todo el territorio poblado por los wichís. Por esta razón, en la lengua wichí encontramos mayormente préstamos del español y del quichua, y muchas palabras cognadas con las otras lenguas de la familia lingüística mataco-mataguayo, y algunas con el toba (Censabella, 1999; Vidal y Nercesian 2009a; Montani 2017)
} 
grupos indígenas, tal como lo indican algunos prestamos del quichua, desde el comienzo del siglo XIX las interacciones de los wichís con actores foráneos no dejan de intensificarse. Primero con los criollos que vinieron hacia la zona en búsqueda de mejores pastizales por su ganado, luego con los misioneros anglicanos que instalaron sus misiones a las orillas del Bermejo y Pilcomayo y, de poco a poco, con una vasta gama de organizaciones non gubernamentales (ONG) e instituciones públicas activas en el campo del desarrollo. La presencia continua y creciente de nuevos actores y la necesidad de instaurar con ellos un dialogo son dos de los principales factores que, sumado al factor religioso, han contribuido a la difusión de la lengua española entre los wichís. Hoy en día, si bien su lengua es una de las lenguas indígenas más vitales en el país y que un gran numero de sus hablantes son monolingüe -por la mayoría los niños en edad preescolar y los ancianos (Terraza, 2009)- también es cierto que gran parte de los wichís hablan y entienden el castellano, viviendo "entre dos lenguas" (Grosjean 1982). ${ }^{5}$

\section{Hacia una etnografía de las motos y de su gente}

Para hacer una etnografía de las motos es imprescindible hacer una etnografía de sus dueños o, mejor dicho, de los usuarios de estas motos, definiendo el contexto social y espacial en el que se ha desarrollado el trabajo de campo ${ }^{6}$. Efectivamente, como señalado previamente, estudiar el léxico mecánico con grupos wichís situados en distintas partes de la región chaqueña puede llevar muy probablemente a resultados algo diferentes en razón de la diversidad interna de la lengua wichí y en función de las distintas influencias ejercidas por factores foráneos y por actores no wichís. Por eso, es necesario precisar que las palabras de las motos y las reflexiones que de esas resultan y que serán expuestas de aquí en adelante solamente son pertinentes si se estudia el sector noroeste del departamento Ramón Lista, que se incluye en la zona mejor conocida en la provincia de Formosa como el "extremo oeste".

Más precisamente, el trabajo de campo ha sido desarrollado en el marco del curso de formación profesional para "Auxiliar mecánico de motos - Mecánico de motos" organizado en las localidades de María Cristina y Lote 8 por el Núcleo de Formación Permanente y Formación Profesional de la Provincia de Formosa. ${ }^{7}$ Según Gustavo,

\footnotetext{
5 Hay que destacar que el nivel de conocimiento y de habla de la lengua castellana depende de la zona de estudio. En la zona del Pilcomayo, me animo a afirmar que la mayoría de los wichís pueden tener una discusión en castellano, aunque se manejen con más soltura en su lengua. Entre los hablantes bilingües, la mayoría han sido escolarizados recién a partir de los últimos años de la década de 1990 cuando se instalaron en la zona las primeras escuelas.

${ }^{6}$ El trabajo de campo al cual estoy haciendo referencia tuvo lugar en los meses de junio y julio del año 2017.

7 Tal como se puede leer en el sitio internet de la provincia, esta institución "organiza y ofrece cursos de corta duración o trayectos formativos organizados por módulos que facilitan al estudiante transitar según su propia disponibilidad de tiempo, acreditando saberes
}

miembro de la Delegación Zonal de María Cristina, "el objetivo del curso de mecánica es que los alumnos sean socialmente activos. Digamos que se persigue a la vez la escolarización y la formación de los adultos, buscando profesionalizarlos (...). Se trata de inculcarles la cultura del trabajo, para que sepan hacer algo que les permita desenvolverse en la sociedad" (entrevista del 15/06/2017). Esta ultima afirmación confirma la imagen de "vagos" que las instituciones tienen de los wichís y otros habitantes de la zona, por lo que se necesita "inculcarles la cultura del trabajo" menospreciando sus otras competencias.

Si estas afirmaciones hacen recordar a la "integración social del indio" perseguida por el indigenismo latinoamericano, hay que remarcar que este curso es también una forma para descongestionar otras formaciones brindadas precedentemente, por las cuales hoy en día la oferta sobrepasa por mucho a la demanda, siendo el mercado laboral local -que de por sí ya es reducido y además está saturado- limitado casi únicamente a empleos públicos. Asimismo, nacido de una demanda por parte de la comunidad, este curso remplaza las formaciones en carpintería que hasta hace poco eran brindadas por los organismos estatales en esta misma localidad como una forma de dar una salida laboral a los jóvenes recién egresados del colegio. Este cambio de formación técnica, de la carpintería a la mecánica de motos, atestigua los cambios que los wichís están viviendo y la evolución de sus aspiraciones y perspectivas en el mundo del trabajo.

El curso para devenir "Auxiliar mecánico de motos Mecánico de motos" es abierto a todo el mundo: ${ }^{8}$ los habitantes indígenas de la zona, en su gran mayoría wichís, y los criollos, tanto hombres como mujeres. A pesar de eso, casi todos los alumnos inscriptos al curso se reconocen como wichís y muy pocos como criollos. Asimismo, una sola mujer asiste las clases de mecánica en María Cristina, mientras ninguna participa del curso en Lote $8 .{ }^{9}$ En cambio, se puede decir que la edad es más bien heterogénea, porque los alumnos tienen entre $18 \mathrm{y}$ 45 años. Los jóvenes son los que más asiduamente asisten a las clases, mientras los más ancianos son los que más faltan, probablemente porque tienen otros quehaceres o toma temporariamente algún empleo. Asimismo, hemos remarcado una cierta heterogeneidad socio-profesional que caracteriza a los alumnos: por la mayoría son jóvenes recién salidos del colegio, otros son adultos desocupados

y desarrollando capacidades que le permitan insertarse laboralmente o fortalecer, y/o actualizar sus conocimientos para mejorar su nivel de desempeño y remuneración en los lugares de trabajo o generar sus propios proyectos productivos ya sea de manera individual o asociativa" (Núcleo de Formación Permanente y Formación Profesional de la Provincia de Formosa, 2017).

8 El único requisito para acceder a los cursos ofrecidos por el mencionado Núcleo es haber terminado la escuela primaria.

9 Es difícil fijar un numero preciso, aunque sea indicativo, de participantes a los dos cursos porque muchos se inscriben, pero no van a las clases, sino puntualmente. En línea general, estimo que medianamente hay 20 alumnos que participan de manera activa y continua. 
o trabajan como jornaleros, y algunos pocos son MEMA o tienen otro tipo de empleo público. ${ }^{10}$ Sus diversas trayectorias hacen que tengan diferentes niveles de conocimiento sobre el funcionamiento y la reparación de las motos.

Si es legitimo afirmar que ninguno de los jóvenes que asisten las clases de mecánica tiene un buen conocimiento de la composición y el funcionamiento de un motor, hay que reconocer que a la hora de inscribirse casi todos poseían sin embargo una moto. Eso no quiere decir que cada uno tenga su propia moto, sino que al menos comparten una con sus parientes. ${ }^{11}$ Así, por ejemplo, algunos comparten una moto entre 8 hermanos, otros poseen 3 motos entre 13 parientes en la casa y algunos otros tienen 2 motos para 3 hermanos y hermanas. ${ }^{12}$ Asimismo, es importante poner en evidencia que no es cierto que "tener una moto" signifique siempre "tener una moto que funcione". Muchas veces están paradas; otras tantas, anduvieron, pero ya no andan, son las motos "en espera" a las que me refería antes. Lo que en cambio sí es cierto, es que tanto a María Cristina como a Lote 8 "tener una moto" es considerado por los padres como una inversión. Comprar una moto para los hijos implica que sigan con los estudios y vayan a completar su formación al terciario con el objetivo de obtener rápidamente un empleo y un salario que permitirá recuperar el gasto $y$, posiblemente, ayudará así a la familia. ${ }^{13}$

Es importante operar una distinción entre el curso que tiene lugar en María Cristina y el de Lote 8. Si bien estas dos localidades se sitúan sobre el área que fue asignada por el Estado nacional a que se fundara una colonia agrícola al comienzo del siglo $\mathrm{XX}$, se diferencian según tres variables principales que tienen cierto efecto sobre la manera en que se desarrolla el léxico mecánico. Una primera variable tiene que ver con la historia de las dos localidades en relación con la presencia de habitantes criollos. En María Cristina, la centralidad política y social es eminentemente Wichí, dado que en el pueblo muy pocos son los criollos que viven, concentrándose en una especie de barrio separado de los Wichís. En cambio, la población de Lote 8 está repartida de manera más equitativa, caracterizándose a lo largo de estos últimos años por una fuerte concentración de población criolla

\footnotetext{
10 La sigla MEMA corresponde a Maestro Especial Modalidad Aborigen, actividad que se desarrolla en el marco de la Educación Intercultural Bilingüe (EIB).

11 Para una explicación detallada de la extensión referencial del termino "parientes" en la lengua wichí, cf. el excelente trabajo de Palmer (2005).

12 La única condición que los jóvenes entrevistados han destacados con respecto al compartir las motos con sus parientes es que estos últimos "no tomen" o que "no sean tomadores". A la luz de los problemas de alcoholismo que caracterizan a esta región, el hecho de tomar representa un riesgo muy probable, o una casi certeza, de un choque, y por ende de un daño que implicará un gasto importante.

13 Mientras en María Cristina hay un segundario desde 2000 y en Lote 8 desde 2007, el único terciario del departamento se encuentra a más de 60 kilómetros de estas localidades, en el pueblo de El Potrillo.
}

en buena medida proveniente del vecino Paraguay. La presencia más o menos consistente de criollos juega un rol fundamental sobre el vocabulario utilizado durante los cursos y sobre el repertorio lingüístico de los jóvenes que participan de ellos.

Una segunda variable para tener en cuenta a la hora de estudiar las palabras de la moto en la lengua indígena, es la cohabitación en la misma localidad de comunidades wichís de grupos dialectales distintos. Los jóvenes que atienden el curso de mecánica en Lote 8 hablan diferentes dialectos: algunos tienen padres y abuelos que pertenecen a grupos montaraces que recién se han instalado en ese lugar en los años 1970, otros vienen de las comunidades situadas a la orilla del antiguo curso del río Pilcomayo, antes de que se desplazase por las inundaciones de la década de 1990. En cambio, si bien en el pueblo de María Cristina cohabitan varias comunidades, todas son originarias del río, habiéndose progresivamente juntado en esta zona después de las inundaciones de los años 1990. Aquí la influencia de los misioneros anglicanos fue mucho más contundente, gracias a la instauración de una escuela donde los niños wichís aprendían la lengua castellana. Por eso, si su escaso uso en Lote 8 depende de los distintos dialectos, en María Cristina la mayoría de los alumnos maneja las dos lenguas sin mayores dificultades. En este sentido, la historia del poblamiento de esos lugares marca las culturas locales e influye sobre los dialectos y el volumen del repertorio lingüístico de sus habitantes.

Por fin, la última variable que importa considerar es el contexto donde concretamente se desarrollan los cursos. En María Cristina las clases de mecánica se desarrollan dentro de un edificio recientemente construido, que tenía función de escuela para la comunidad de San Miguel, hasta que ésta se alejó del pueblo para instalarse a unos kilómetros. Este edificio es ahora la sede de la Delegación Zonal del área y las clases tienen lugar por la mañana en un aula que cuenta con luz eléctrica y es utilizada para otras actividades por la tarde. En cambio, tal como lo muestra la Figura 1, en Lote 8 el curso se hace en el viejo edificio de la escuela, una pieza que estaba en estado de casi abandono hasta que los alumnos no la han limpiado y reacomodado. Pero como no hay ventanas ni puertas ni luz eléctrica, esta pieza sigue siendo por las noches un lugar donde los jóvenes se reúnen a emborracharse. De esa manera, si el contexto edilicio de María Cristina se presta a las clases teóricas y favorece un tipo de aprendizaje "escolar", en Lote 8 es más bien difícil crear un ambiente propicio a la transmisión escolar de un saber mecánico.

\section{Desarmando motos, moldeando palabras}

Se trata ahora de hacer unos comentarios sintéticos sobre el desenvolvimiento de las clases, tomando en cuenta las dificultades estrechamente vinculadas a la 


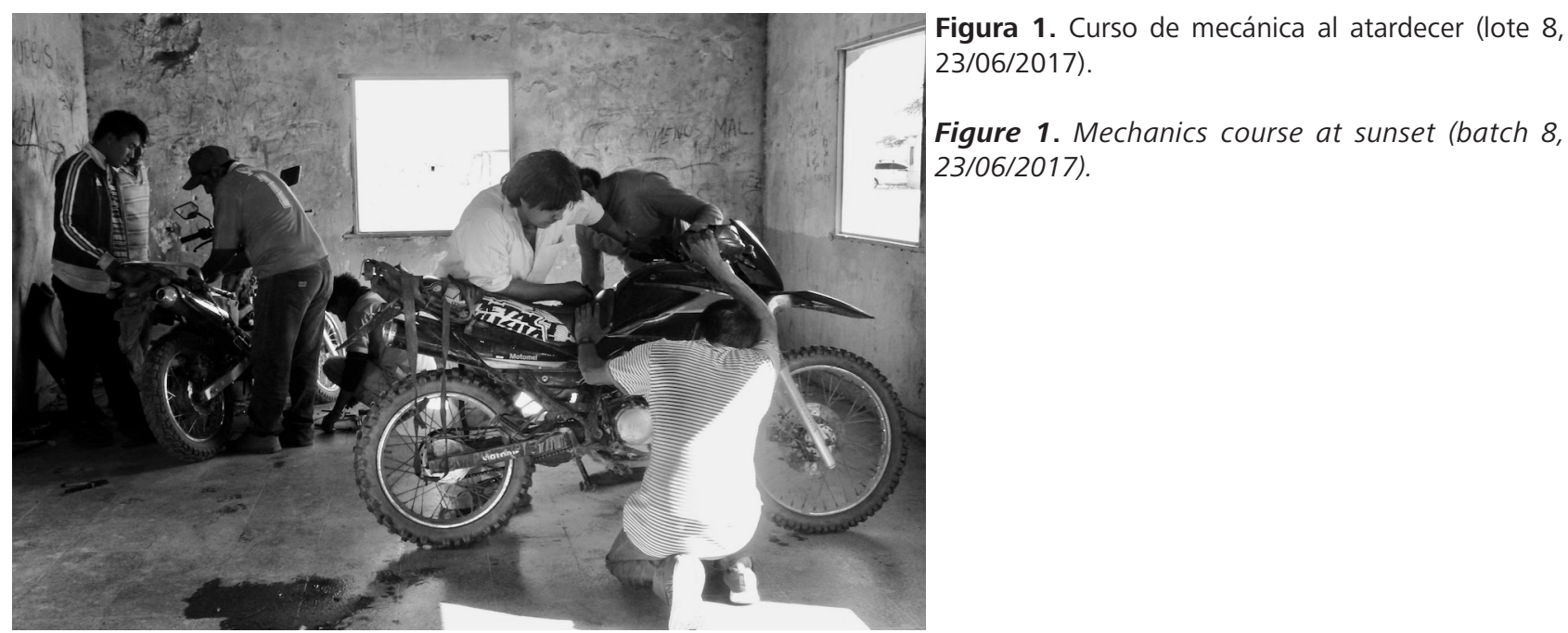

enseñanza de la mecánica y su traducción en la lengua wichí. Los problemas más difíciles para la traducción de la mecánica parecen ser dos. Primero, la necesidad de importar -léase, traducir por préstamos- ciertos conceptos físicos elementales, como fuerza, potencia o corriente. Segundo, la dificultad de los hablantes de encontrar un referente para estos conceptos. Por eso, me focalizaré sobre la figura del profesor que se ocupa de este curso, tratando de entender cuál y cuánta influencia ejerce su pedagogía sobre el proceso de aprendizaje de los jóvenes wichís y sobre la constitución de su repertorio lingüístico con respecto a las motos. Además, me baso sobre la constatación de que "las formas de enseñar y aprender son posiblemente aun más significativas que los contenidos porque a través de ellas se transmiten implícitamente la naturaleza de las relaciones entre educador y educando y los conceptos que la misma cultura guarda acerca del proceso de formación de la persona en su integridad" (Pérez et al., 2012, p. 17).

Cuando realicé mi trabajo de campo el profesor era un joven de 21 años, la misma edad, o incluso menos, que sus alumnos. Originario de la ciudad de Ingeniero Juárez, distante de 150 kilómetros de los poblados en cuestión, Gastón hacia constantemente el esfuerzo de adaptar sus clases para que resultasen interesantes e inteligibles para sus alumnos, mayoritariamente wichís. Según sus palabras, "a parte que no me siento cómodo con eso, no sirve que escriba al pizarrón el jargón técnico para indicar las distintas piezas de un motor y tampoco sirve que utilice los vocablos técnicos. Los chicos se aburren y muchas veces no me entienden porque eso no tiene ningún sentido para ellos y tampoco quiere decir algo en su idioma" (entrevista del 09/06/2017). Tal como lo afirma el profesor, frente a la dificultad encontrada a la hora de explicar teóricamente los tiempos de un motor y los distintos procesos de combustión, de distribución y de transmisión que ocurren para que una moto ande, él había preferido emprender una pedagogía más bien aplicada, intentando aprender algo de la lengua wichí para facilitar de ese modo los intercambios con los alumnos.

Así es como las clases para devenir "mecánico" o "auxiliar mecánico" se volvían cada vez menos un curso formal, donde el profesor se distancia de sus alumnos, para tomar rápidamente la forma de un aprendizaje horizontal y compartido. De esa manera, se aprende del ejemplo real y concreto del otro, en este caso el profesor deja su rol académico para asumir un rol más bien de guía, porque los jóvenes wichís aprendan mirando y mirándolo. Se trata de un tipo de aprendizaje que pasa más por la observación que por la explicación. ${ }^{14}$ La atmosfera es muy similar a la de los múltiples talleres mecánicos que se encuentran en los pueblos aledaños. El ruido de los motores, las manchas de aceite en el suelo, el olor a nafta, las numerosas cajas de útiles casi indistinguibles incluso para los ojos más expertos, el ritmo de los martillos y los gritos de acierto o de yerro son solamente algunos de los tantos elementos que caracterizan estas clases de mecánica.

Es apostando, comprometiéndose y probando como los alumnos logran entender el funcionamiento de un motor, sin necesariamente saber repetir al dedillo los procedimientos escritos por el profesor al pizarrón, sin tener quizá conciencia ni conocimiento de lo que significa realmente "combustión" o "transmisión" y lo que implican concretamente estos procesos. Es más que nada a través de los intentos y de la práctica que los jóvenes logran aprender, tocando con la mano un motor desastrado, un pistón roto, un aro doblado. ${ }^{15}$ De esa manera, la pedagogía adoptada por el profesor adhiere a

\footnotetext{
14 Eso mismo suele pasar, por ejemplo, con los jóvenes rom/sinti, cuya experiencia didáctica ha sido objeto de algunas publicaciones en antropología de la educación cf. Gomes A.M.R. 2017.

15 Los alumnos aprenden desarmando primero sus propias motos y luego la de algún pariente al que le piden una "participación", o sea un pago más bien simbólico, aunque en dinero, con el que se compra una merienda para todos los que asisten al curso. Sin embargo, cuando hay que hacer el pedido de un repuesto, romper algo o, en lo peor de los casos, tirarlo, siempre se contacta el dueño de la moto, quien tiene que tomar la decisión final.
} 
la pedagogía wichí que se distingue por dos características principales. Por un lado, es sumamente "experiencial", basada en la vivencia más que en la adquisición teórica de habilidades y conocimientos académicos. Por otro lado, está basada en el equilibrio de la relación entre profesor y alumno, que mantienen una relación simétrica y horizontal, "prestando igual importancia a la capacidad y el conocimiento del educador, así como a la voluntad del que quiere aprender" (Pérez et al., 2012). Este tipo de aprendizaje es más beneficioso para los jóvenes wichís para quienes el léxico mecánico, de otra manera tan lejano e incomprensible, se vuelve más fácil para entender, porque se inserta en su cotidianidad.

Además de eso, es importante destacar que el aprendizaje del funcionamiento de las motos se acompaña de la progresiva constitución de un léxico mecánico que se enriquece cada vez más de nuevos términos en lengua castellana a la vez que integra palabras propias de la lengua wichí hablada por los alumnos. Si por un lado eso les permite apropiarse culturalmente de este nuevo objeto, por el otro, es importante que aprendan los términos en castellano para referirse a las piezas del motor. De hecho, cuando los jóvenes wichís tienen que arreglar sus motos y necesitan ciertos repuestos, no pueden recurrir a los misioneros, los lingüistas o los antropólogos interesados en aprender su lengua, sino que deben interactuar con mecánicos y kiosqueros criollos que rara vez conocen alguna palabra en wichí o, en el mejor de los casos, manejan sólo los rudimentos de esa lengua.

Tal como resaltaron varios jóvenes wichís, el léxico mecánico que aprenden a lo largo de las clases es una herramienta aun más importante de las capacidades técnicas que desarrollan. Así lo explica Leandro: "hay que conocer los nombres de los repuestos para entenderse con los kiosqueros. Si te vas al kiosquero sin saber que pedir o cual es el nombre exacto de la pieza que necesitás, no te va a vender nada o peor aún te va a vender cualquier cosa. Y es peor todavía porque no hablan todos iguales. Por más que uno conozca los nombres de los repuestos, hay veces que los kiosqueros les nombran diferente que nosotros. Lo único que te puede servir es conocer el nombre exacto de la pieza que necesitás. De otra manera, uno se equivoca y le tocará hacer otro viaje" (entrevista del 30/06/2017). De esa manera, aprender las palabras de la moto les permite no solamente ahorrar dinero arreglando ellos mismos su moto, sino también corregir a los mecánicos que trabajan en los pocos y carísimos talleres que hay en los dos poblados. ${ }^{16}$

\section{En el taller, arreglando, reparando y hablando de motos}

Se trata ahora de hacer una etnografía de las motos a

\footnotetext{
${ }^{16}$ En total, hay 3 talleres informales en María Cristina y uno solo en Lote 8 . Los precios de las reparaciones son altos y muchas veces los arreglos son solo provisorios.
}

partir de las palabras y de los usos que de ellas hacen los jóvenes wichís. No obstante, si bien este recorrido tiene como objetivo final identificar las palabras de la moto, es imprescindible considerar a su "antepasado": la bicicleta. En efecto, desde la llegada de los misioneros anglicanos en la década de 1910 -y a parte los tractores, los camiones y las $4 \times 4$ de estos últimos- las bicicletas han representado el único medio de transporte accesible para los wichís hasta la década del 2000. Se utilizaban para ir de una aldea a otra, para visitar a los parientes que vivían en sitios más alejados, para ir a cazar, a pescar o incluso a recolectar frutos, para buscar leña. En fin, desde su temprana introducción hasta su definitiva consagración, la bicicleta ha sido integrada a todas actividades sociales y económicas de los wichís.

La adopción de la bicicleta ha implicado para los wichís una pequeña revolución de los medios de transporte, acelerando los desplazamientos y repercutiendo en el repertorio de actividades y en la organización de la vida doméstica (Montani, 2017). Su éxito ha implicado una progresiva apropiación cultural de este vehículo, que se manifiesta entre otras cosas en su traducción a la lengua wichí ${ }^{17}$. Así pues, el término "bicicleta" se traduce a veces por el vocablo wänlhäj que literalmente significa ñandú o suri (Rhea americana). Esta extensión semántica del nombre del suri se justifica por el hecho que al igual que este animal, que cuando corre para escapar se mueve zigzagueando, dejando una huella sinuosa y ondulada sobre el suelo arenoso del Chaco, la bicicleta deja también una huella que jamás es recta, porque el conductor tiene que evitar espinas, pozos, madrejones, animales y toda otra clase de obstáculos.

Aparte de wän/häj, otro vocablo que los wichís utilizan para nombrar a la bicicleta es yelataj o el término compuesto lataj-chinaj. La palabra yelataj o su aféresis lataj significa "caballo", mientras -chinaj se traduce como "hierro" o, más en general, se usa para indicar que tal u tal objeto es hecho de algún tipo de "metal". En este caso, la traducción wichí de "bicicleta" combina un nombre de material con un nombre de animal. Por lo tanto, combina una extensión semántica del animal, motivada por una similitud funcional -ambos se montancon una especificación del material. Dejando de lado todo intento de asimilación a un animal y sin hacer referencia a la etimología de la palabra en castellano -doble (bis), ciclos (ruedas) - se da prioridad al material del objeto. De esa forma, latah-chinaj o "caballo de hierro", "caballo de metal" y wänlhäj o "suri", son hoy en día los dos términos que más se utilizan para nombrar a la bicicleta en lengua wichí. ${ }^{18}$

\footnotetext{
17 Esta apropiación lingüístico-cultural se refiere no solamente el nombre de la bicicleta, sino a la traducción de casi la totalidad de sus partes (ver Montani, 2017, p. 323).

18 A esos dos términos, R. Montani (2017) agrega el préstamo "wisikleta" y "lëwote" (p. 323)
} 
Si bien la moto constituye una revolución aún más importante de las bicicletas porque achica todavía más los tiempos de desplazamiento a la vez que agranda las distancias transitables, no existe por ahora una traducción común en lengua wichí para el termino "moto" -sin tomar en cuenta el préstamo moto o mutu- y tampoco para "motor". Tan cierto es que en los diccionarios de la lengua wichí, tanto en los antiguos (Hunt, 1937) como en los más recientes (Claesson, 2008; Lunt, 2016), no se incluye ningún termino que haga referencia o traduzca el termino español "moto". A pesar del uso excepcional que se hace actualmente de este medio de transporte y su popularización masiva hacia los rincones más recónditos del Chaco, a parte los prestamos, la única palabra de origen wichí que se utiliza para nombrar a la moto es -wute, "montura", que siempre se acompaña de su partícula posesiva. Si bien se trata de una palabra wichí, no se puede decir que haya sido pensada ni adaptada para referirse específicamente a las motos, porque también se la utiliza para aludir a los caballos o a otros animales de monta y, también, ocasionalmente, a las bicicletas.

Esta falta de palabras wichís para traducir el termino "moto" que vemos en los diccionarios, es confirmada por mi trabajo de campo. ${ }^{19}$ El bisílabo "moto" es de lo más simple de pronunciar por los wichís, por lo que lo han incorporado como préstamo, moto. Eso no quiere decir que las motos estén culturalmente menos apropiadas o menos difundidas que las bicicletas. Al revés, esta falta de un nombre wichí especifico para la moto sugiere a mi parecer que se trata de un objeto que posiblemente escapa a las traducciones que hacen referencia al entorno ambiental, porque se integra con más dificultades en el sistema de semejanzas que permite "animalizar" por ejemplo las bicicletas. Al mismo tiempo, es verdad que no hay un neologismo en lengua wichí para "moto" porque se trata de otro momento en la historia de esta lengua y porque el termino castellano se presta perfectamente a ser tomado como un préstamo. Por esta razón, es más pertinente volcar nuestra atención a las piezas de la moto para las que, en cambio, abundan los términos en lengua wichí.

\section{Dando forma a un nuevo léxico mecánico}

Durante de las clases de mecánica los alumnos wichís aprenden un vasto léxico mecánico que se compone de los términos técnicos que necesitan para identificar cada una de las piezas de una moto y, a la vez, de los que sirven para nombrar llaves, tubos o martillos

\footnotetext{
19 Asimismo, nos importa subrayar que -contrariamente a lo que podía pensar- los alumnos que participan de los cursos de mecánica reconocen las diferencias entre los múltiples modelos de motos que circulan en la región, pero no hacen ninguna distinción a la hora de nombralos en wichí. Ya sea que se trate de una Motomel modelo "Skua", de una Mondial, de una Honda modelo "XR", de una Appia modelo "Leyenda", o de una Corven modelos "Hunter" o "Triax", siempre se emplean los mismos vocablos "-wute" o "moto" para nombrarlas en wichí.
}

en lengua castellana. La importancia de este ultimo conjunto de términos resulta de la importancia de poseer herramientas, lo que constituye un problema para los aspirantes mecánicos ${ }^{20}$. Por un lado, muchas veces no conocen el término técnico que define a cada una de las herramientas necesarias; por el otro, no tienen los medios para comprarlos. Participar de las clases se vuelve pues una posibilidad de aprender $\mathrm{y}$, al mismo tiempo, de acceder a las herramientas que no se tienen en la casa.

El léxico mecánico que se integra de a poco al vocabulario bilingüe de los jóvenes alumnos wichís -que como dije, son bilingües wichí-castellano- está entonces compuesto tanto por los términos que nombran cada una de las piezas de la moto como por aquellos que nombran las herramientas que sirven para repararla. Unas y otras tienen a la vez un nombre oficial, técnico, que es muy poco utilizado en esta zona del Chaco donde se habla prevalentemente una versión local del castellano $y$, justamente, otro nombre en "criollo" o "chaqueño" -como se le dice al dialecto en la región. ${ }^{21}$ En este sentido, el aprendizaje por parte de los jóvenes wichís se complejiza, sumando un estrato lingüístico a un vocabulario ya de por sí complicado. Tal como lo explica Demetrio, "hay veces que uno solo encuentra el problema de la moto, pero como no sabe el nombre de la pieza que está fallando, se le hace imposible cambiarla. O hay veces que uno lo sabe, pero no logra hacerse entender por los kiosqueros porque no es el término correcto" (entrevista del 22/06/2017). Por esta razón, jóvenes como Demetrio o el citado Leandro tienen que aprender a la vez los términos técnicos, que les permiten ir a comprar los repuestos o formular pedidos a distancia, ${ }^{22}$ y el nombre en criollo para entenderse o dar indicaciones a los mecánicos de los talleres locales.

Como ejemplos de esos estratos lingüísticos que es necesario manejar para hablar de un motor, cito aquí una serie concisa de piezas acompañadas por los términos técnicos correspondientes y los nombres que se les da localmente. Así pues, el "conjunto de clutch", mejor conocido como "manzana de embragues", es habitualmente definido en la zona como "canasta de

\footnotetext{
20 Las herramientas necesarias para arreglar un motor son generalmente muy caras, en 2017, una caja de herramientas costaba alrededor de los \$6 000. Dada las dificultades económicas en las que viven la mayoría de las familias de esos jóvenes, por lo general las herramientas se comparten entre varios, así como se comparten las motos o la nafta. También, a veces, se fabrican esos útiles.

21 En términos generales, se podría analizar esta alternancia entre términos castellanos y criollos según la diferencia de códigos lingüísticos introducidos por Bernstein (1964). Llegado el caso, el castellano ocuparía el lugar del código ampliado, y el criollo lo de código restringido.

22 Desde la zona del Pilcomayo, es común que se busquen o se pidan repuestos en Bolivia, donde son más baratos que en los grandes asentamientos de la región del lado argentino, como Santa Victoria Este o Ingeniero Juárez. Además, hay que subrayar que es imposible comprar repuestos en los kioscos de María Cristina o de Lote 8, porque nadie tiene.
} 
embrague", lo que implica que los jóvenes wichís aprendan tres diferentes términos para identificar a una única parte. De la misma manera, para indicar el "amortiguador" (termino técnico) se utiliza "botella" (termino criollo). Asimismo, para hablar del "descompresor del motor" se privilegia la expresión "bomba subterránea", haciendo referencia a los brotes del motor. Otras veces se usa el nombre de una sola pieza para nombrar un conjunto, como es el caso del término criollo "biela" que substituye el termino técnico "cigüeñal". O se tiende a generalizar entre piezas similares: los "retenes" y todo otro tipo de guarnición se conocen como "gomitas". Por fin, en cuanto a las herramientas es interesante observar que localmente no se hace ningún tipo de distinción entre los diferentes martillos, y se suele llamar "llaves" a los "tubos".

Para nombrar las piezas de la moto, cuando existe la posibilidad de simplemente traducir un término castellano con un termino indígena equivalente desde el punto de vista semántico, los wichís no buscan crear neologismos. Por eso, por ejemplo, el "piñón loco" simplemente se traduce como "piñón amukwe", que en wichí también significa "delincuente", en el sentido de inmoral, que no respeta las reglas. Otros términos hacen referencia a su material, tal como se utiliza chinaj (hierro) para hablar de las bicicletas. En este sentido se usa poho, "estar roto en dos partes", o wasi, "estar hecho pedazos", para nombrar las luces de la moto. En efecto, al estar hechas de un plástico que mal se adapta a las intemperies propias del Chaco y rompiéndose cada vez que alguien se cae a causa de los madrejones de arena y de barro, el nombre en lengua wichí evidencia la fragilidad del material con el cual están hechas las luces. Pero según los jóvenes entrevistados, estas no son las únicas palabras que se utilizan para indicar las distintas luces de la moto. También se puede utilizar el término täte-lhoy que significa "los globos oculares de alguien".

Otras veces, los wichís hacen énfasis en la finalidad que tienen las distintas piezas y herramientas. Por esta razón, el termino compuesto layalh-cha, donde layalh se traduce como "su aire" o "su aliento", mientras -cha corresponde a "instrumento". Así pues, layalh-cha substituye el término español "inflador". De la misma manera, en lengua wichí se identifica el "tanque" como nasta-hi, que literalmente significa "el recipiente (-hi) de la nafta". Otro ejemplo similar de un término que hace hincapié en la finalidad del objeto más que en su material es el compuesto castellano "guardabarros", que es muy parecido al término wichí lakalä̈-pot, donde lakalä̈, que literalmente significa "sus piernas", aquí refiere a "sus ruedas" y -pot representa "tapa", como la de la hoya para cocinar. Para terminar, según los jóvenes wichís la "Ilave" que sirve para hacer arrancar la moto, se la indica a través del término polisémico "lacha", un artefacto que también sirve para "hacer un guiso" o "para limpiar la casa".

Si algunos de los términos wichís que traducen nombres castellanos de piezas de la moto hacen referencia a los materiales con que están hechas y otros se refieren a sus funciones, buscando algunas consonancias con objetos propios del espacio doméstico, más todavía son los vocablos que aluden a partes de animales y humana. No se trata de una característica propia de la lengua wichí y tampoco confirma una equiparación entre seres humanos y animales aplicada a los artefactos mecánicos, aun que esta hipótesis sea posiblemente correcta. A mi parecer, el intento de encontrarles y darles nombres de partes del cuerpo animal -y humano- a las partes de las motos demuestra más que nada una aproximación cultural a las motos por parte de los wichís. Se trata de encontrar elementos propios de su entorno social y ambiental que, por analogía, permitan volver inmediatamente inteligibles ciertos términos que de otra manera escapan a su lógica y no tienen ningún sentido.

Así pues, cada moto tiene "sus cuernos", lech'ul, para referirse al manubrio; "sus piernas", lekalä, para hablar de las ruedas; "sus tripas", lekä̈ley para indicar las cámaras; "sus brazos", lakweyey para indicar las suspensiones delanteras o barrales; ${ }^{23}$ la "piel de su pierna", lëakälä-t'äj, para la cubierta; y su proprio "esqueleto", "sus huesos", Ihiley, vocablo que sirve para indicar el cuadro. Todos estos términos son muy similares a los que se utilizan para nombrar a las partes de la bicicleta (Montani 2017). La animalización/humanización de las motos no es simplemente relativa a sus elementos inmediatamente visibles, sino que se aplica igualmente al mismo motor. Los jóvenes wichís señalan que cada moto tiene "su carne" o "su pulpa", t’isän, término que hace referencia a la caja del motor, donde se encuentra el corazón pulsante de la moto y que hay que tratar con mucho cuidado a la hora de desmontar para repararlo (Figura 2). En fin, vale subrayar que el funcionamiento de algunas piezas de las motos, como las suspensiones delanteras, o su forma fálica, como la del pistón, poseen a los ojos de los wichís una carga sexual muy fuerte que suscita risas o vergüenza al momento de indicarlos.

Esta dinámica que consiste a familiarizar lo ajeno -o sea tomar como referencia las cosas que mejor se conocen desarrollando analogía con lo más cercano- para poder aceptarlo en su mundo es muy similar a lo que pasa en el ámbito del parentesco (Palmer 2005; Montani 2017). Tal como en otros contextos interétnicos, los términos polisémicos (Brown y Witkowski 1983) y la invención de expresión figurativas similares (Brown y Witkowski 1981) se multiplican. Eso puede también manifestarse a la hora de identificar ciertos ruidos de las motos. Los wichís, sobre todo los menos jóvenes que participan a las clases de mecánica, prestan mucha atención a los distintos sonidos emitidos por los motores que andan bien y los que no. Es

\footnotetext{
${ }^{23}$ Señalo que algunos jóvenes wichís para indicar las suspensiones delanteras también utilizan el término mälhewek que significa "entero, completo" para referirse a esta pieza fundamental de la moto que no sirve si no se dispone de todas las partes que las componen.
} 


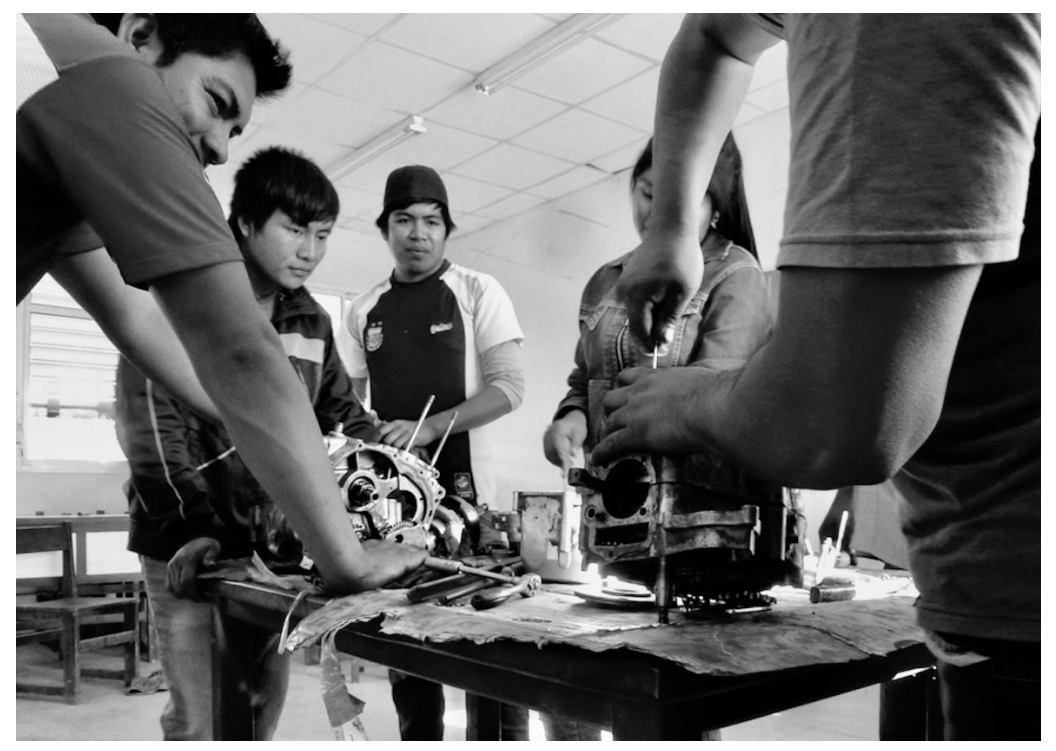

Figura 2. Arreglando la caja del motor, t'isän (María Cristina, 09/06/2017).

Figure 2. Fixing the engine box, t'isän (Maria Cristina, 09/06/2017).

como si estuviesen escuchando los sonidos de un animal del monte durante una noche de caza para interpretarlos y anticipar sus movimientos. Por esta razón, en las clases suelen acelerar muchas veces los motores, escuchando su lap'ak, "su voz", "su bramido", como si se tratase de un jaguar o de un toro escondido en el monte, con el objetivo de encontrar el origen del problema.

Por último, es importante mencionar que los términos utilizados por los wichís para indicar algunas partes del motor demuestran una comprensión intima de su funcionamiento y de la organización inmutable que es necesaria para que una moto arranque. Por eso, se habla de aros- wet, el "lugar de los aros" para indicar las ruedas, y de válvulas-wet, el "lugar de las válvulas", para indicar el cigüeñal. En los dos casos, se hace uso del término wet que tiene diferentes significados entre los cuales se destaca lo de "morada" en el sentido del lugar que le es propio a alguien o algo. Del mismo modo, para indicar al caño de escape, el término correspondiente en lengua wichí es tutsaj-näyij, donde tutsaj quiere decir "humo" mientras - näyij es un adverbio que significa "hace un poco de tiempo" o "hace poco tiempo". Se trata de un término también utilizado para traducir la "chimenea" de una estufa, que según Cesáreo se explica por el hecho de que "el humo siempre se va por ahí, pero no queda, tiene que salir rápido según un camino recto y sin parar demasiado" (entrevista del 22/06/2017).

\section{Conclusión}

En algún momento del pasado no muy lejano, los términos anatómicos wichís se han ampliado para incluir varios artefactos -o las partes de esos artefactos- de la cultura material introducida por actores foráneos en el Chaco formoseño. Tal como lo señala Kaith Basso respecto a la integración de los automóviles en el mundo y en la lengua apaches (1967), la extensión de los términos anatómicos primero a las bicicletas, y luego a las motos, ha sido extremadamente funcional para que incorporen el mundo wichí. No solamente porque ha facilitado la comunicación significativa sobre un objeto totalmente extraño en un marco de referencia familiar, sino también, porque ha vuelto innecesario lanzarse en un sistema de categorías y clasificaciones complicadas, que puede ser incómodo incluso para los no wichís.

Todavía, si bien pueda resultar temprano para ciertas afirmaciones, me atrevo a decir que, en buena medida, los términos que los wichís utilizan para nombrar las partes de la moto, y aun más del motor, son prestamos castellanos. Eso no quiere decir que sea una lengua escasamente inventiva, sino que, puestos en frente a la dificultad de encontrar traducciones adaptadas y de inmediata comprensión, para los wichís resulta más sencilla la adopción de términos castellanos. La presencia frecuente de prestamos castellanos entre las "palabras de las motos" es común cuando no es posible, o no conviene, o resulta demasiado complejo encontrar una traducción, aun más cuando se trata de vocabulario técnico (cf. el trabajo de Monino (1970) sobre los Ngbaka de África central).

De esa manera, los prestamos mecánicos integran un corpus ya vario y consistente de otros prestamos que se van multiplicando a medida de la intensificación de los contactos entre los wichís y actores foráneos (Vidal y Nercesian 2009b). Eso atestigua de una situación común por muchas otras lenguas indígenas latinoamericanas (Brown 1994) y de la porosidad lexical no solamente del wichí sino también del castellano.

En fin, la aculturación léxica de los wichís, o más bien su aprendizaje de las palabras de las motos, atestigua de la complejidad del mundo interétnico que toma forma y sentido en el Chaco en la medida que elementos de 
las diversas culturas se entremezclan. Si espero haber logrado demostrar que la lengua se ha vuelto una herramienta imprescindible para estudiar los cambios culturales, sin embargo, en este articulo solo he abordado estos cambios desde la perspectiva wichí desconociendo la perspectiva criolla cuyo análisis merecería una atención particular.

París, 3 de abril 2020

\section{Agradecimientos}

Agradezco a Rodrigo Montani y Zelda Franceschi por su voluntad de avanzar en el conocimiento del mundo wichí a través el estudio de su lengua y, particularmente, por haber aceptado que un geógrafo opine sobre esa materia. Gracias también al profesor y a los jóvenes wichís que me dieron la posibilidad de acompañarlos durante las clases del curso de mecánica. En fin, agradezco el apoyo y el financiamiento del proyecto ANR JJCC 201720 "Mécaniques Sauvages. Le fait mécanique dans les sociétés amérindiennes du Chaco et de I'Atacama". Este articulo se ha desarrollado en el marco de una investigación doctoral financiada por la Universidad Paris 1 Panthéon-Sorbonne.

\section{Bibliografía}

Alvarsson, J-A. (1988). The Mataco of the Gran Chaco: An Ethnographic Account of Change and Continuity in Mataco Socio-Economic organization. Uppsala: Acta Universitatis Upsaliensis vol. 11.

Basso, K.H. (1967). Semantic aspects of linguistic acculturation. American anthropologist, 69(5), 471477.

Bernstein, B. (1964). Elaborated and Restricted codes: their social origins and some consequences. American Anthropologist, 66(6), 55-69.

Braunstein, J. (2006). El signo del agua: Formas de clasificación étnica wichí. En I. Combès (Ed.), Definiciones étnicas, organización social y estrategias políticas en el Chaco y la Chiquitania (pp. 145-156). Santa Cruz: IFEA, SNv \& El País.

Brown, C.H. (1994). Lexical Acculturation in Native American Language, Currrent Anthropology, 35(2), 95-117.

Brown, C.H. (1999). Lexical acculturation in native American languages. New York: Oxford University Press.

Brown, C.H. y Witkowski, S.R. (1981). Figurative Language in a Universalist Perspective. American Ethnologist, 8(3), 596-615.
Brown, C.H. y Witkowski, S.R. (1983). Polysemy, Lexical Change and Cultural Importance, Man, 18(1), 72-89.

Censabella, M. (1999). Las lenguas indígenas en la Argentina. Buenos Aires: EUDEBA.

Córdoba L., Bossert F. y Richard N. (Eds.). (2015). Capitalismo en las selvas: Enclaves industriales en el Chaco y Amazoníaindígenas (1850-1950). San Pedro de Atacama: Ediciones del Desierto.

Dorais, L-J. (1970). L'acculturation lexicale chez les esquimaux du Labrador. Langages, 18, 65-77.

García, S.S. (2016). Aculturación léxica en El Gran Nayar: presencia del cora en el español del siglo XVIII. Temas Antropológicos. Revista Científica de Investigaciones Regionales, 38(2), 19-44.

García, S.S. y García Molina, A. (2016). Estrategias de aculturación léxica del náayeri o cora: un estudio de disponibilidad léxica. Boletín de Lingüística, 45-46, 134-152.

Gomes, A.M.R. (2017 [1998]). "Vegna che ta fago scriver". Etnografia della scolarizzazione in una comunita di Sinti. Roma: CISU.

Grosjean, F. (1982). Life with Two Languages: An Introduction to Bilingualism. Cambridge: Harvard University Press.

Krohn, H.S. (2016). Aculturacion lexica en bribri. Estudios de Lingüística Chibcha, 35, 75-93.

Monino, Y. (1970). Dérivation, composition et emprunt dans le vocabulaire des techniques Ngbaka-Ma'bo (République Centrafricaine). La Linguistique, 6, 117-146.

Montani, R. (2017). El mundo de las cosas entre los wichís del Gran Chaco. Un estudio etnolingüístico. Cochabamba: Itinerarios.

Núcleo de Formación Permanente y Formación Profesional de la Provincia de Formosa, 2017, https://www.formosa. gob.ar/noticia/20788/12/ofertas_de_los_nucleo_de_ educacion_permanente_y_formacion_profesional_en_ la_provincia_.

Palmer, J. (2005). La buena voluntad wichí: Una espiritualidad indígena. Buenos Aires: Grupo de trabajo Ruta 81.

Pérez, E. et al. (2012). Educación entre los Wichí. N'ochufwenyajay ta iyej Wichi. Salta: SMA Ediciones.

Pina-Cabral, J. (2014). World: An anthropological examination (part 1). HAU: Journal of Ethnographic Theory, 4(1), 49-73. 
Susnik, S. (1969). Chamacocos 1 Cambio cultural. Asunción: Museo etnográfico Andrés Barbero.

Terraza, J. (2009). Grammaire du wichí: Phonologie et morphosyntaxe. Tesis de doctorado,Universidad de Québec à Montréal. MS.

Vidal, A. y Nercesian, V. (2009). Loanwords in Wichi, a Mataco-Mataguayan language of Argentina. En M.
Haspelmath y U. Tadmor (Eds.). Loanwords in the world's languages: a comparative handbook (pp.1015-1034). Berlin: Mouton de Gruyter.

Vidal, A. y Nercesian, V. (2009b). Estudio del léxico wichí (mataco-mataguaya): Aportes al conocimiento de algunas situaciones de contacto y desplazamiento lingüístico en el Chaco argentino. Cuadernos interculturales, 12, 141158. 